\title{
Study on Characteristics of Flow-induced Vibration (FIV) Induced by Gas-liquid Two- phase Flow in the Conveying Pipe
}

\author{
W Chunsheng, L Zejun, Z Yan, S Qiji* \\ School of Petroleum Engineering, Northeast Petroleum \\ University, Daqing, Heilongjiang Province, China
}

\begin{abstract}
The multi-physics coupling study is carried out by considering the fluctuation of the interphase dynamic interface and its flow characteristics as well as the mechanical properties of the solid structure of the pipeline. By capturing the dynamic interface between gas and liquid, calculating its flow characteristics, combined with the time-history displacement and amplitude of the characteristic monitoring point and the axis of the pipeline, the FIV response characteristics of the basin in the S-type conveying pipe are analyzed. The results show that the dominant area of single gas phase or single liquid phase is not easy to deform and vibrate. The transition and persistence of the flow pattern in the mixed turbulent flow area and the weak regular fluctuation of the flow field in the pipe stimulate the pipe wall to cause stress reaction, leading to the time-history vibration with great difference in the pipe.
\end{abstract}

\section{INTRODUCTION}

As a classical subject with parametric dynamic systems, the study of dynamic characteristics of divergence instability, single-mode and coupled-mode flutter, bifurcation and chaos in the fluid conveying pipes is also the most attractive and practical issue in the field of fluid-solid coupling research that is really related to industrial safety and development. Hydrodynamic force that is applied on the structures, pipes and various objects will produce destructive vibrations, and may lead to the acoustic and noise problems in the industrial machinery, which is known as Flow-Induced Vibration (FIV). FIV will hinder the smooth operation of engineering equipment and may result in serious consequences. In recent years, the subject has become increasingly important in the engineering industry for single-phase and multiphase flows as well as various flow directions including external and internal flows[1]. CFD technique shows good potential in FIV research [2-5].

Regarding the study of the FIV problem, domestic and foreign scholars pay more attention to the vortex-induced vibration and galloping phenomenon of the bluff body structure caused by the fluid flow around. Mature research methods have been developed with in-depth research results obtained for the cylindrical vibration of different shapes, the multi-degree-offreedom column flutter, the vibration characteristics of the platy structure and other related issues under the high Reynolds number. There are relatively few studies on the basin induced pipeline instability flutter in the long-diameter pipelines with thin structures. In recent years,

ॠCorresponding Author: sunqiji@nepu.edu.cn 
many scholars have begun to pay attention to the FIV problem in pipes. Ter Hofstede et al. [6-8] studied FIV in a nuclear fuel rod using single phase RANS $\mathrm{k}-\omega$ SST and RSM models by coupling CFD and solid mechanics model. Liu, Y., Miwa et al. [9-11] conducted an experimental study on FIV in vertical and horizontal directions under an elbow with a diameter of $52.5 \mathrm{~mm}$ and an inner diameter of $90^{\circ}$, to study the fluctuating forces induced by internal two-phase flow on pipe bends. By separating the natural frequency of test section from the predominant frequency of excitation forces, the dynamic force signals were obtained directly from force sensors. A total of 36 tests were carried out to cover bubbly, slug, churn and annular flows. Gayet et al.[12] studied the flow induced forces in a horizontal $180^{\circ}$ pipe bend of 4" (101.6 mm) diameter using CFD. The VOF model was used for the two-phase flow modelling, while LES for turbulence modeling to predict slug flow at the superficial liquid and gas velocities of $2.4 \mathrm{~m} / \mathrm{s}$ and $2.2 \mathrm{~m} / \mathrm{s}$ respectively. Mazdak Parsi et al. simulated the vertical air-water churn flow using MultiFluid VOF model, and obtained the data such as phase distributions, mean void fractions, and average void fraction time series from the CFD simulation These data were compared with the previously obtained experimental Wire Mesh Sensor (WMS) data. The simulation shows that MultiFluid VOF model can capture different physical scenarios related to the churn flow [13-14]. J.-L. Riverin et al. observed severe vibrations in a small piping system that consists of two elbows and straight sections of a Ushaped structure and is subject to the air-water internal two-phase flow. They carried out an experimental study to study the excitation mechanism of vibration control. The vibration response, excitation forces and fluctuating characteristics of the two-phase flow were measured under extensive flow conditions. The results show that the observed vibration is caused by the resonance between the periodic momentum flux fluctuation of the two-phase flow and the first mode of the pipe system[15-18].

Therefore, this study is aimed at the flow-induced vibration (FIV) phenomenon induced by gas-liquid two-phase complex turbulent flow in the S-type conveying pipe, the multi-physics coupling study is carried out by considering the fluctuation of the interphase dynamic interface and its flow characteristics as well as the mechanical properties of the solid structure of the pipeline. By capturing the dynamic interface between gas and liquid, calculating its flow characteristics, combined with the time-history displacement and amplitude of the characteristic monitoring point and the axis of the pipeline, the FIV response characteristics of the basin in the S-type conveying pipe are analyzed.

\section{PHYSICAL MODEL}

Consider the length-diameter ratio and laying mode of the gas-liquid two-phase fluid conveying pipes in the far-off oil and gas engineering block, and establish the physical model of the S-type fluid conveying pipes into the export fixed branch, as shown in Figure 1. This paper focuses on the relationship between the gas-liquid two-phase flow characteristics and the instability vibration characteristics of the pipeline, so the model is adjusted and optimized: The actual operation of the far-off engineering block includes the facilities such as insulation layer, the anti-corrosion layer and the weight coating. For layers and other facilities, this paper ignores the specific pipe wall components, only considers the wall thickness and applies steel material to cover the pipe wall subdivision facilities; because the two-phase viscosity has a weak influence on the pipe vibration, the gas phase working medium in this paper is air, and the liquid phase working medium is liquid water.

Figure 1 is a schematic diagram of the physical model of the S-type fluid conveying pipes for transporting gas-liquid two-phase fluid. The detailed piping system parameters and fluid property parameters are shown in Table 1. 
The monitoring points $\mathrm{A}, \mathrm{B}$, and $\mathrm{C}$ are set at the riser pipe of the pipeline in the fluid conveying pipes, and the time-history displacement under the action of the two-phase flow is solved. The coordinates of each monitoring point are: monitoring point $\mathrm{A}(2.17,9.75)$, monitoring point $\mathrm{B}(-0.67,4.98)$, monitoring point $\mathrm{C}(-2.98,1.62)$.

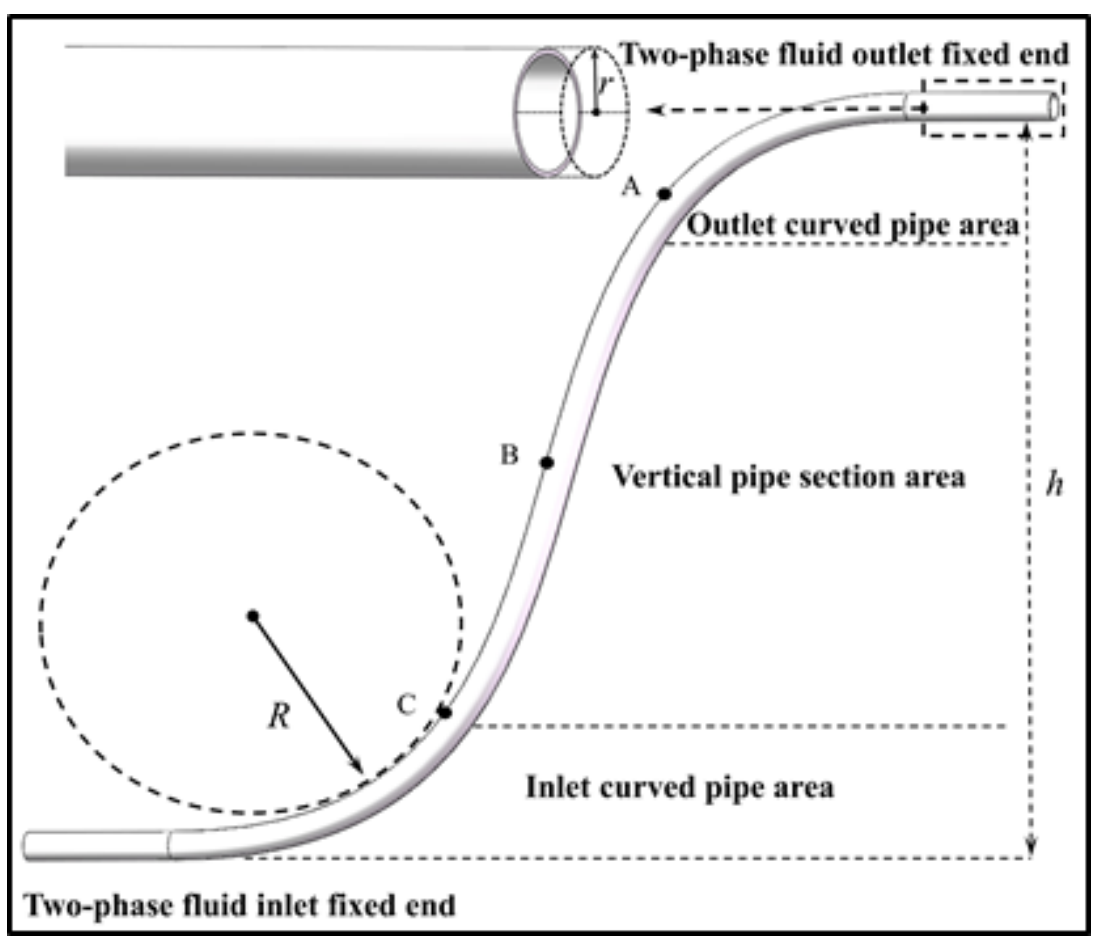

Figure 1: S-type-fluid conveying pipes FIV model

Table 1. The S-type-fluid conveying pipes correlation parameter

\begin{tabular}{|c|c|c|c|c|c|c|}
\hline model & $\begin{array}{l}\text { physical } \\
\text { parameters }\end{array}$ & value & $\begin{array}{l}\text { material } \\
\text { parameters }\end{array}$ & value & $\begin{array}{l}\text { Fluid } \\
\text { parameters }\end{array}$ & value \\
\hline \multirow{4}{*}{$\begin{array}{l}\text { Two- } \\
\text { dimensional } \\
\text { pipeline } \\
\text { model }\end{array}$} & $L$ length $[\mathrm{m}]$ & 30 & $\begin{array}{l}\text { Density } \\
{\left[\mathrm{kg} / \mathrm{m}^{3}\right]}\end{array}$ & 7850 & $\begin{array}{l}\text { Liquid velocity } \\
{[\mathrm{m} / \mathrm{s}]}\end{array}$ & 0.50 \\
\hline & \multirow{3}{*}{$\begin{array}{l}R \text { Curvature } \\
\text { radius[m] } \\
r \text { Outer } \\
\text { diameter[m] } \\
D \text { Wall } \\
\text { thickness[m] }\end{array}$} & 0.6 & $\begin{array}{l}\text { Young's modulus } \\
{[\mathrm{Pa}]}\end{array}$ & $200 \mathrm{e} 9$ & $\begin{array}{l}\text { Gas velocity } \\
{[\mathrm{m} / \mathrm{s}]}\end{array}$ & 1.20 \\
\hline & & 0.55 & Poisson's ratio $v$ & 0.30 & Gas-liquid ratio & 0.30 \\
\hline & & 0.025 & & & $\begin{array}{l}\text { Density } \\
{\left[\mathrm{kg} / \mathrm{m}^{3}\right]}\end{array}$ & 1013 \\
\hline
\end{tabular}




\section{METHODOLOGY}

Computational Fluid Dynamics technique has been used in the present study to extract fluctuating flow properties due to multiphase flows. Since the focus of the study is the FIV response characteristics of the basin in the S-type conveying pipe are analyzed, the multiphase volume of fluid(VOF) method is utilized to track the interface between liquid and gas phases.

\subsection{Continuity, momentum and volume fraction}

In the VOF method, a single set of momentum equations is shared by phases (here, air and water) and the volume fraction of each phase is tracked through the computational domain. The governing equations for the VOF model are given below[19]:

Continuity:

$$
\frac{\partial \rho}{\partial t}+\nabla \cdot(\rho \bar{u})=0
$$

Momentum:

$$
\frac{\partial(\rho \bar{u})}{\partial t}+\nabla \cdot(\rho \bar{u} \bar{u})=-\nabla P+\nabla \cdot \mu\left(\nabla \bar{u}+\nabla \bar{u}^{T}\right)+F
$$

The surface tension force in Eq. (2) is represented by $F$. The surface tension force is expressed as a volume force and is added to the momentum equation as a source term.

To track the interface between phases a volume fraction continuity equation for one of the phases (water in this case)is solved along with the above equations:

$$
\frac{\partial \alpha_{1}}{\partial t}+\vec{u} \cdot \nabla \alpha_{q}=0
$$

where subscript q represents each phase component.

Air volume fraction is obtained from the relation

$$
\sum_{q=1}^{2} \alpha_{q}=1
$$

The properties appearing in the transport equations are determined by the presence of the component phases in each control volume. For example, the density is considered to be:

$$
\rho=\sum_{q=1}^{2} \alpha_{q} \rho_{q}
$$

The surface tension effects between liquid water and air has been considered by using the continuum surface force (CSF) model. According to this model, the volume force is added to the momentum source as 


$$
F=\sigma\left(\frac{\rho k_{1} \nabla s_{1}}{1 / 2\left(\rho_{I}+\rho_{g}\right)}\right)
$$

where, $\sigma$ is the surface tension coefficient, and $k_{1}$ is the surface curvature of the liquid droplet defined in terms of the divergence of the unit normal, and is given by,

$$
k_{1}=\nabla \cdot \overline{n_{1}}
$$

The unit normal vector, $n_{1}$ is calculated from the local gradients in the surface normal at the interface as,

$$
\overline{n_{1}}=\frac{\nabla s_{1}}{\left|\nabla s_{1}\right|}
$$

Wall adhesion effects are accounted for by adjusting the surface curvature near the wall, where gas-liquid interface meets the solid wall. The local curvature of this interface is determined by the contact angle, $\theta_{w}$, which represents the angle between the wall and the tangent to the interface at the wall. The surface normal vector at the wall is given by,

$$
\bar{n}=\overline{n_{w}} \cos \theta_{w}+\overline{t_{w}} \sin \theta_{w}
$$

where, and are the unit vectors normal and tangential to the wall, respectively.

\subsection{Turbulence model}

The mixture turbulence model is the simplified extension of the single phase $k-\epsilon$ model. In this model, it is deemed that the mixture properties and mixture velocities could adequately capture the main features of turbulent flow.

The mixture turbulent kinetic energy $k$ is given by[20]:

$$
\nabla \cdot\left(\rho_{m} \vec{v}_{m} k\right)=\nabla \cdot\left(\frac{\mu_{t, m}}{\sigma_{k}} \nabla k\right)+G_{k, m}-\rho_{m} \epsilon
$$

The mixture energy dissipation rate $\epsilon$ is given by:

$$
\nabla \cdot\left(\rho_{m} \vec{v}_{m} \epsilon\right)=\nabla \cdot\left(\frac{\mu_{t, m}}{\sigma_{\epsilon}} \nabla \epsilon\right)+\frac{\epsilon}{k}\left(C_{1 \epsilon} G_{k, m}-C_{2 \epsilon} \rho_{m} \epsilon\right)
$$

where, the mixture density and velocities are given by:

$$
\begin{gathered}
\rho_{m}=\sum_{i=1}^{N} \alpha_{i} \rho_{i} \\
\bar{v}_{m}=\frac{\sum_{i=1}^{N} \alpha_{i} \rho_{i} \bar{v}_{i}}{\sum_{i=1}^{N} \alpha_{i} \rho_{i}}
\end{gathered}
$$


The turbulent viscosity, $\mu_{t, m}$ is computed from:

$$
\mu_{t, m}=\rho_{m} C_{\mu} \frac{k^{2}}{\epsilon}
$$

And the production of turbulent kinetic energy, $G_{k, m}$ is computed from:

$$
G_{k, m}=\mu_{t, m}\left(\nabla \bar{v}_{m}+\left(\nabla \bar{v}_{m}\right)^{T}\right): \nabla \bar{v}_{m}
$$

\subsection{Mesh generation}

As shown in Fig. 2(a), the 8th iteration boundary layer is used to smoothly transition to the internal grid of the two-phase flow basin at the boundary layer of the pipe conveying fluid, and the sharp corner is processed by the split method. The split angle range is $240 \mathrm{deg} \sim 100 \mathrm{deg}$, the maximum layer reduction is 2 , the maximum depth is 16 , the tensile factor at the boundary is 1.2 , the minimum thickness of the first boundary layer is about $0.02 \mathrm{~mm}$, and the maximum thickness of the boundary layer is about $0.48 \mathrm{~mm}$.

Structured meshing is adopted for two-phase flow basins and pipeline walls in S-type pipe conveying fluid. The basin and pipeline walls are comprehensively calibrated by fluid dynamics and solid physics, and the ultra-fine grid of 10-4 orders of magnitude is divided. model. The pipeline wall and the basin area are divided by free triangle structure. The grid curvature factor is 0.2 , the narrow area resolution is 1 , and the largest unit of the grid is $0.8 \mathrm{~mm}$. The boundary layer is the same as the near wall boundary layer, and the number of iterations is 8. The processing depth is 16, as shown in Figure 2(b).

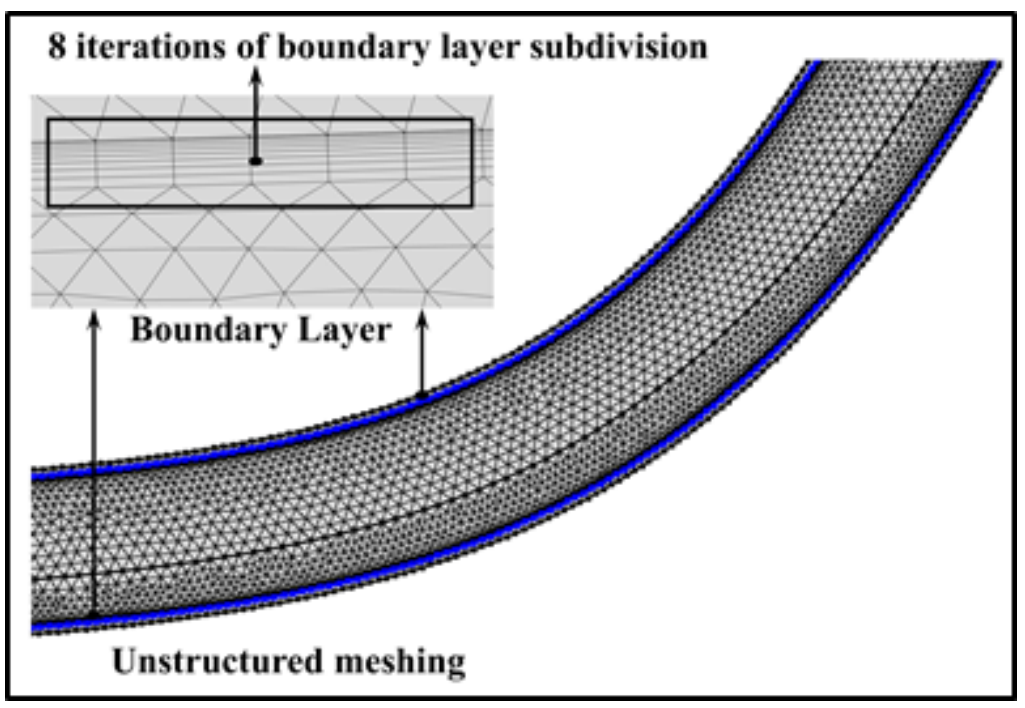

(a) Near wall unstructured mesh 


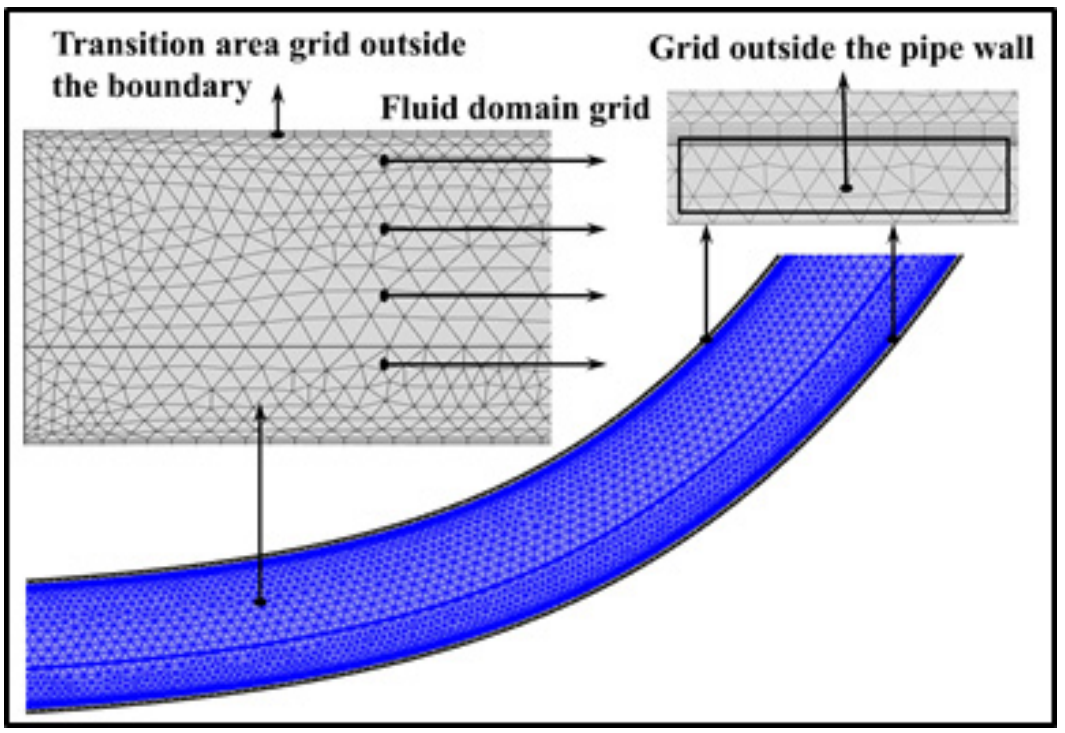

(b) Structured mesh of basin and pipe wall

Figure 2: Schematic diagram of two-phase flow basin, pipe wall and near wall interface

\section{RESULTS AND DISCUSSION}

In this paper, the numerical analysis of the induced vibration of gas-liquid two-phase flow in S-transport pipeline is carried out. Based on the finite element method of partial differential equation, COMSOL.Multiphysics-5.3a platform was used in coupling calculation of twophase dynamic interface capture equation, multi-phase basin motion equation and multiphysics field of pipeline solid-structure mechanics equation. The dynamic nonlinear laws such as gas-liquid interface fluctuation, fluid velocity field distribution, pipeline stress distribution and time-history displacement are analyzed in detail. The response relationship between the two-phase fluid flow characteristics and the flow-induced vibration characteristics of the pipeline is discussed.

\subsection{Gas-liquid dynamic interface capturing}

Figure 3 shows the results of the full cycle tracking calculation of the gas-liquid two-phase dynamic interface in the pipeline based on the VOF model. It can be seen from the figure that the interphase dynamic interface of the full-cycle flow process in the S-transport pipeline presents complex disorder fluctuations. The initial stable flow in the basin, the two-phase moving surface quickly begins to weakly fluctuate, enters the riser area, the gas phase components are compressed to cause a surge in momentum, and the liquid-carrying rise begins, forming a short annular flow, and then the dynamic interface fluctuates increasingly violent, and bubbly flow and liquid phase slug appear, gas and liquid continue to rise and the dynamic interface tends to stabilize cross flow. That is to say, in the complex conversion and continuous flow of the tube in the disordered fluctuation of the two-phase dynamic interface, the transmission pipeline will be unstable and flutter. 


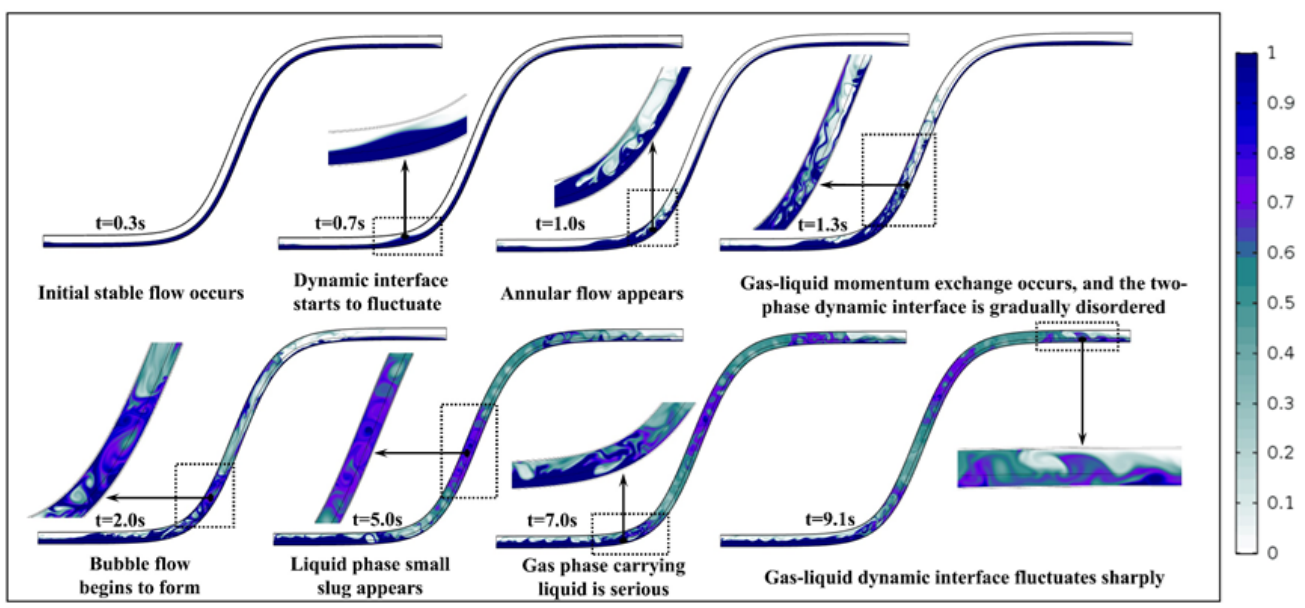

Figure 3: Gas-liquid two-phase periodic dynamic interface tracking in S-type-fluid conveying pipes.

\subsection{Velocity field distribution}

Figure 4 shows the fluid velocity field distribution in the basin based on the S-transport pipeline physical model combined with equation, The full-cycle velocity field shows a weak regular trend. It is consistent with the change law of the two-phase moving surface in the tube. The initial gas-liquid two-phase velocity field is stable, and the fluid enters the riser region through the curved pipeline. The gas phase is compressed to cause a surge in velocity. The momentum exchange with the liquid phase begins to rise with liquid. The fluid impinges on the wall of the pipeline and undergoes energy conversion. At this time, the velocity field exhibits high-speed dynamic fluctuations, and the fluid is subjected to the wall stress in the pipeline that is fixed to the outlet. The centrifugal force generated by the pipeline bending is the fluid. Relative inertia force, the coupling effect of the inertial force and the axial pressure caused by the rotation of the pipe section shows that the $\omega$-type law decreases with the continuous flow and gradually becomes stable and fluctuating. During the full-cycle motion of the fluid in the basin, the above-mentioned weak regularity changes occur due to the restraint of the pipe wall, causing the instability vibration of the pipe conveying fluid.

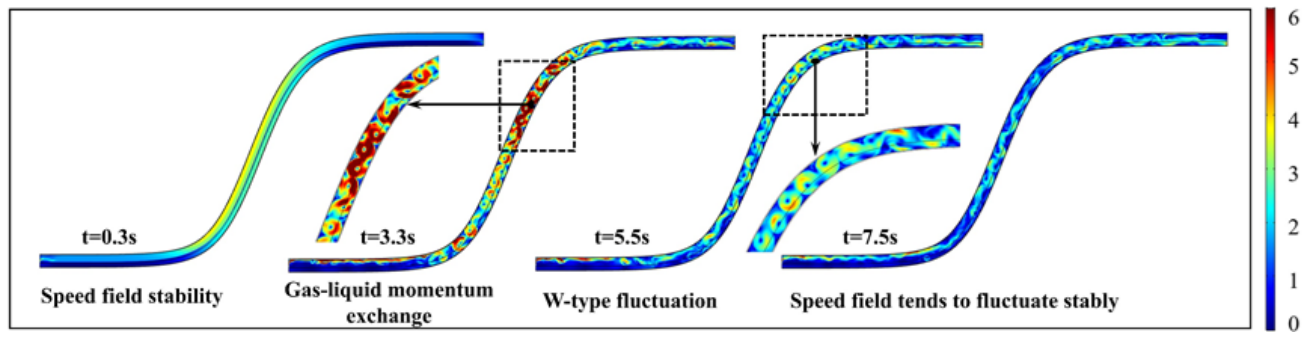

Figure 4: Fluid velocity field distribution patterns at different times. 


\subsection{Pipe wall stress field distribution}

Figure 5 shows the steady stress field distribution curve of the pipeline under the action of two-phase fluid. It can be seen that the solid branch end is higher than the riser pipe section than the curved pipeline area, and full-cycle displacement flutter up and down relative to the axis of the pipeline. Combined with the above description of the two-phase fluid dynamic interface tracking and velocity field, the dynamic interface of the tube rapidly disorder fluctuates after a short time displacement of initial filling, and the velocity field weakly increases regularly. At this time, the pipeline begins to produce displacement instability flutter; With the continuous fluctuation of the gas-liquid dynamic interface and the velocity field gradually tending to reduce the fluctuation law of the $\omega$ type, the pipeline shows a trend of gradual stabilization of flutter.

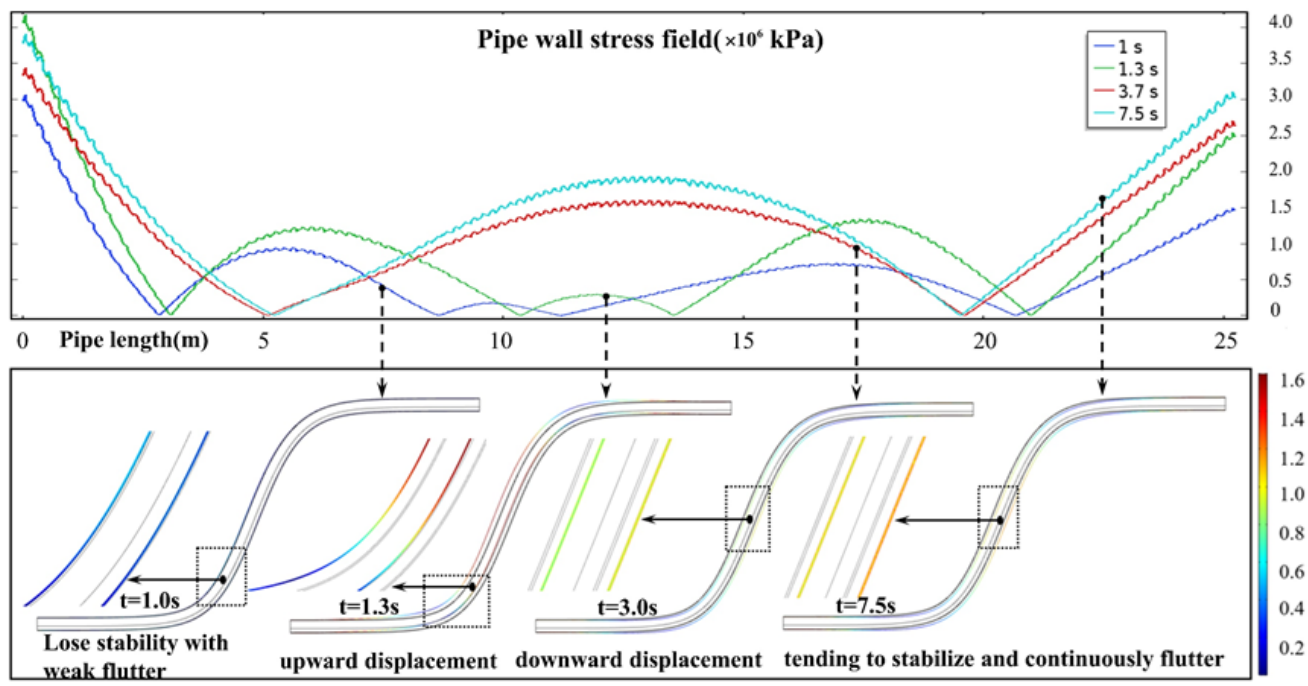

Figure 5: Fluctuations in the stress field of the pipeline wall at different times

Calculate the interphase dynamic interface, tube wall stress field and velocity field distribution of the pipe conveying fluid at 10th time, as shown in Figure 6.

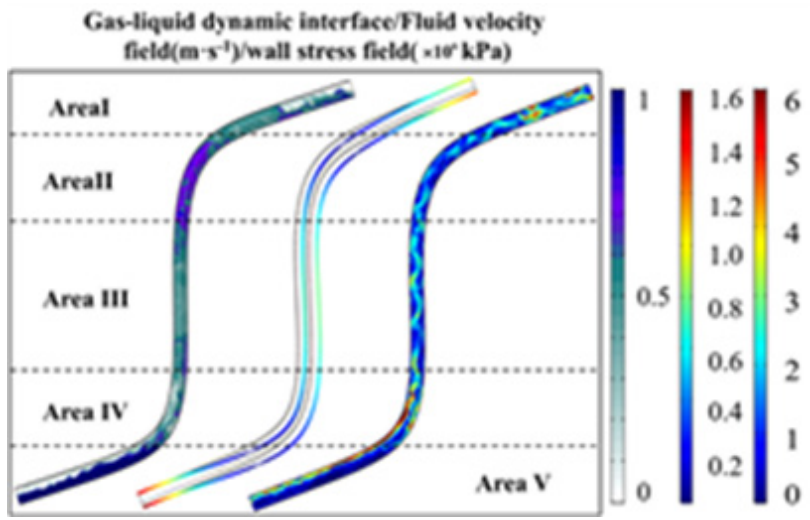

Figure 6: 10s-Multi-field pipeline contrast. 
It can be seen that the fluid conveying pipes exhibits varying degrees of unstable flutter under the induction of different flow characteristics of the two-phase fluid in the basin. The region I is the gas-phase dominant region at the solid branch end of the outlet, and its velocity field has no obvious fluctuation; The area II is the slug flow liquid plug area, where the pressure fluctuation is severe and the velocity field is low; the area III is the slug flow gas plug area, the velocity field exhibits a strong $\omega$-type law; the area IV is the gas-liquid mixed turbulent area, the gas phase velocity field is significantly higher than the liquid phase, and the liquid carrying rises; the region $\mathrm{V}$ is the liquid-phase dominant region, and the velocity does not fluctuate significantly; the I and V stress fields in the region where the inlet and outlet are fixed are higher than the region III is higher than the region at the curved pipeline II IV; therefore, it can be known that the complex turbulent flow pattern and velocity field fluctuations in the basin cause instability vibration of the pipeline, and the single-plug dominated pipeline areas are less prone to vibration.

\subsection{Flutter dynamic tracing}

The time-history displacement curve is calculated for the three monitoring points set in Figure 1, as shown in Figure 7. It can be seen that the initial steady flow of the pipeline does not occur in flutter, and the gas-liquid interaction immediately produces strong displacement. The time-history displacement of the monitoring point $\mathrm{B}$ is significantly higher than that of the curved pipeline, and the monitoring point $\mathrm{A}$ is slightly higher than the monitoring point $\mathrm{B}$. The overall fluctuation law is consistent; as the gas-liquid dynamic interface and velocity field fluctuate gradually stable, it tends to have a stable amplitude of flutter; the single point displacement of monitoring point $\mathrm{B}$ is about $57.5 \mathrm{~mm}$, and the flow is stable at $18.5 \mathrm{~mm}$.

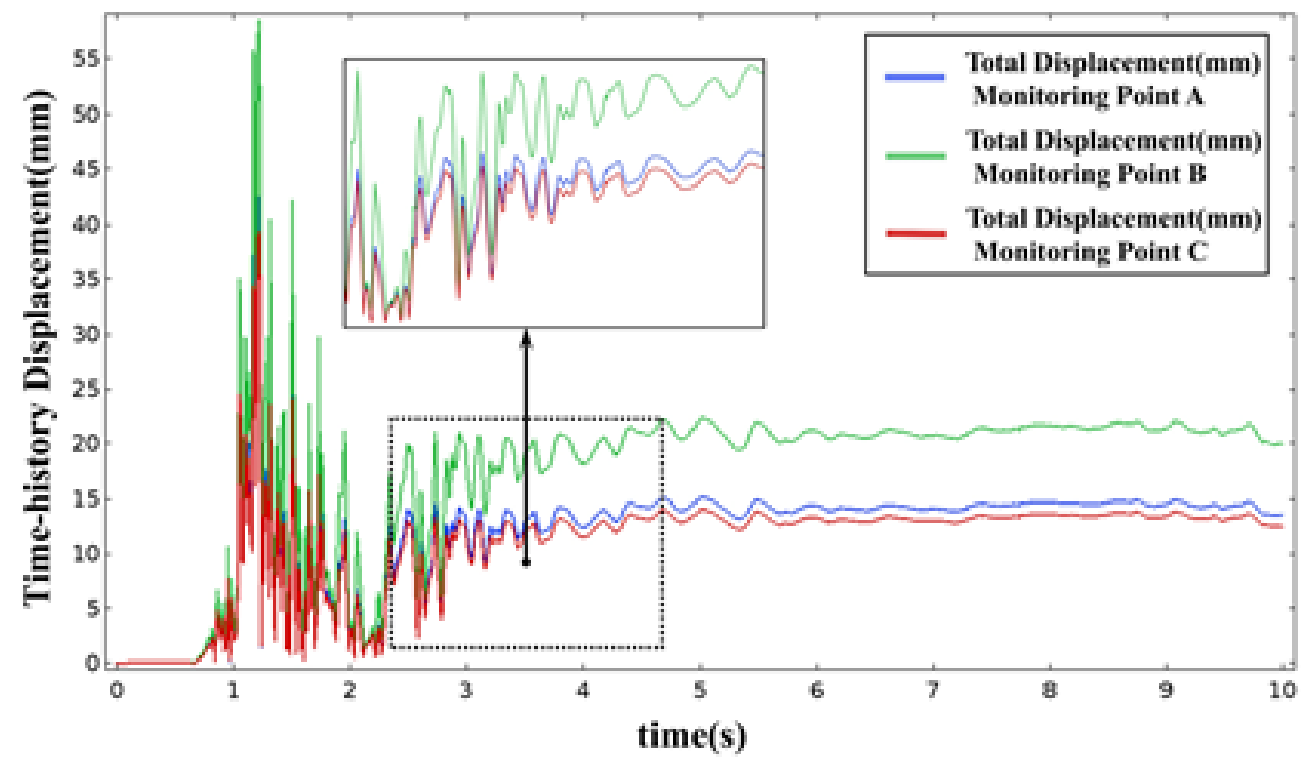

Figure 7: Monitoring point time-displacement curve 
Figure 8 depicts the deformation displacement of the axis of the pipeline at different times. The overall deformation of the pipeline shows a weak normal distribution trend. The deformation displacement of the riser section increases sharply. It can be seen that the displacement at the axis of the conveying pipeline is always in the state of unstable flutter before and after the different time history displacement. After the flow is relatively stable, the single-pass deformation displacement is lower than the previous period.

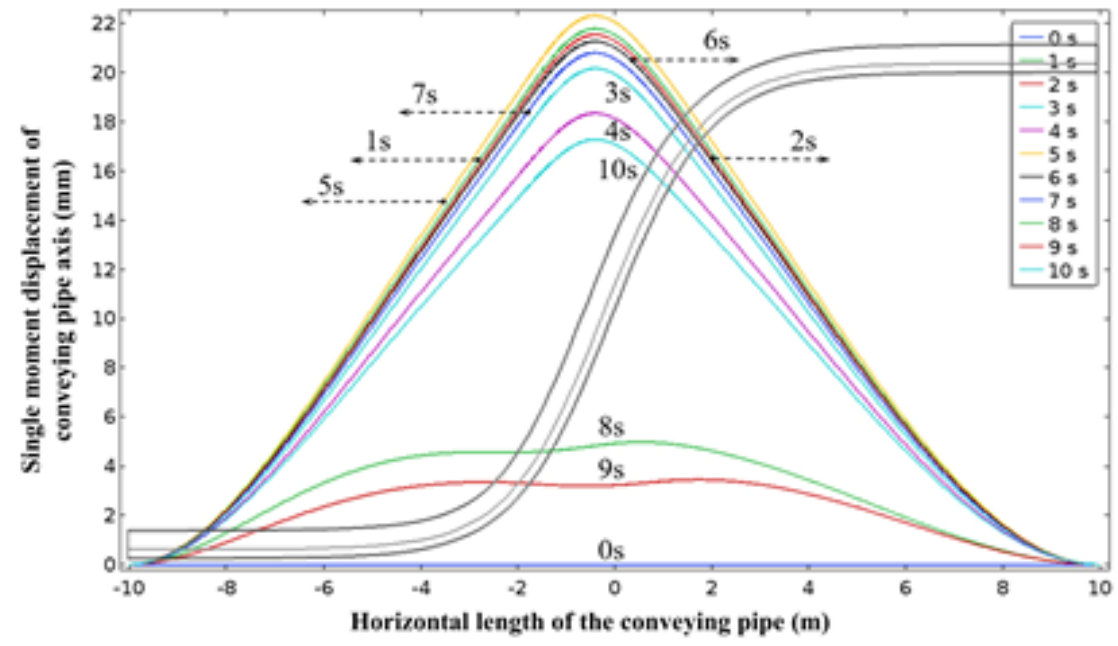

Figure 8: Time-history displacement of pipeline axis

\section{CONCLUSION}

For the longitudinal calculation study on the two-dimensional model of the S-type fluid conveying pipes, the velocity difference between gas and liquid and the coupling effect between the fluid and the pipe wall leads to the complex fluctuation in the interphase dynamic interface in the pipe, which presents the characteristics of complex turbulence such as annular flow, bubble flow and slug flow; the short-term velocity field surge occurs in the early stage of the two-phase fluid flow, and the pipeline suffers significant flutter with high amplitude. Then the velocity field of the fluid in the pipe exhibits a uniformly decreasing $\omega$-shaped fluctuation rule.

During the full-cycle multi-field coupling process of the pipeline, through the stress curve of the pipe wall, the stress value of the fixed end is always higher than that of other regions, and the stress value of the vertical pipe section is always higher than that of the curved pipe, which means that the pipe section between the inlet and outlet bends suffers obvious deformation and instability, and the fixed end has strong stability; at the gas velocity of $1.2 \mathrm{~m} / \mathrm{s}$, the liquid phase velocity of $0.5 \mathrm{~m} / \mathrm{s}$, and the flow parameter of 0.3 gas-liquid ratio, by calculating the time-history displacement of the monitoring point and the axis, the maximum amplitude of the one-way conveying pipe can be obtained as $45 \mathrm{~mm}$, the flow is stable at $15 \mathrm{~mm}$ in the later stage, and the time-history displacement in the vertical pipe area is higher than the curved pipe area, higher than the fixed end. The above calculation and study show that the flutter phenomenon induced by gas-liquid mixed flow is stronger than that of the single gas plug or single liquid plug area. 


\section{SUPPORTING AGENCY}

Natural Science Foundation of Heilongjiang Province: LH2019E017 Study on the non-steady Heat-seepage Coupling Law with Moving Boundaries of SW-SAGD with between Heel and Toe Injection in heavy oil reservoirs.

\section{REFERENCES}

[1] Miwa, S., Mori, M., Hibiki, T., 2015. Two-phase flow induced vibration in piping systems. Prog. Nucl. Energy 78, 270-284.

[2] Ratkovich, N., Majumder, S., Bentzen, T.R., 2013. Empirical correlations and CFD simulations of vertical two-phase gas-liquid (Newtonian and non-Newtonian) slug flow compared against experimental data of void fraction. Chem. Eng. Res. Des. 91 (6), 988998.

[3] Gayet, C., N’Diaye, M., Liné, A., 2013. Behaviour of slug flow and pressure force induced in a spool: Numerical simulation of a taylor bubble flowing in a liquid flow through a spool. In: 16th International Conference on Multiphase Production Technology. BHR Group.

[4] Doussis M P P. The canonical problem of the fluid-conveying pipe and radiation of the knowledge gained to other dynamics problems across Applied Mechanics[J]. Journal of Sound \& Vibration, 2008,310(3):462-492.

[5] Zhu, H., Tang, Y., Wang, J., Tang, L., 2017. Flow erosion and flow induced vibration of gas well relief line with periodic fluctuation of boosting output.J. Loss Prev. Process Ind. $46,69-83$.

[6] Zhu, H., Wang, J., Ba, B., Wu, Z., Wang, W., 2015. Numerical investigation of flow erosion and flow induced displacement of gas well relief line. J.Loss Prev. Process Ind. $37,19-32$.

[7] Ter Hofstede, E., Kottapalli, S., Shams, A., 2017. Numerical prediction of flow induced vibrations in nuclear reactor applications. Nucl. Eng. Des. 319,81-90.

[8] Yih, T.S., Griffith, P., 1968. Unsteady Momentum Fluxes in Two-Phase Flow and the Vibration of Nuclear Reactor Components. MIT Dept. of Mechanical Engineering, Cambridge, Mass. URI: http://hdl handle net/1721.1/61496

[9] Youngs, D.L., 1982. Time-dependent multi-material flow with large fluid distortion. In: Numerical Methods for Fluid Dynamics. Atomic Weapons Research Establishment, Aldermaston.

[10]Liu, Y., Miwa, S., Hibiki, T., Ishii, M., Morita, H., Kondoh, Y., Tanimoto, K., 2012. Experimental study of internal two-phase flow induced fluctuating force on a 90 elbow. Chem. Eng. Sci. 76, 173-187. DOI: https://doi.org/10.1016/j.ces.2012.04.021

[11] Miwa, S., Mori, M., Hibiki, T., 2015. Two-phase flow induced vibration in piping systems. Prog. Nucl. Energy 78, 270-284.

[12] Miwa, S., Hibiki, T., Mori, M., 2016. Analysis of flow-induced vibration due to stratified wavy two-phase flow. J. Fluids Eng. 138 (9), 091302.

[13] Gayet, C., N’Diaye, M., Liné, A., 2013. Behaviour of slug flow and pressure force induced in a spool: Numerical simulation of a taylor bubble flowing in a liquid flow through a spool. In: 16th International Conference on Multiphase Production Technology. BHR Group.

[14] Parsi, M., Vieira, R., Agrawal, M., Srinivasan, V., McLaury, B., Shirazi, S., Schleicher, E., Hampel, U., 2015. Computational fluid dynamics (CFD) simulation of multiphase flow and validating using wire mesh sensor. In:17th International Conference on Multiphase Production Technology. BHR Group,Cannes, France. 
[15] Parsi, M., Agrawal, M., Srinivasan, V., Vieira, R.E., Torres, C.F., McLaury, B.S., Shirazi, S.A., Schleicher, E., Hampel, U., 2016. Assessment of a hybrid CFD model for simulation of complex vertical upward gas-liquid churn flow. Chem. Eng. Res. Des. 105, 71-84.

[16] Riverin, J.L., Pettigrew, M.J., 2007. Vibration excitation forces due to two-phase flow in piping elements. J. Press. Vessel Technol., Trans. ASME 129(1), 7-13.

[17] Pettigrew, M., Taylor, C., 1994. Two-phase flow-induced vibration: An overview (survey paper). J. Press. Vessel Technol. 116 (3), 233-253.

[18] Mishima, K., Ishii, M., 1984. Flow regime transition criteria for upward two-phase in vertical tubes. Int. J. Heat Mass Transfer 27, 723-737.

[19] Ishii, M., Hibiki, T., 2010. Thermo-fluid Dynamics of Two-Phase Flow. Springer, New York.

[20]Launder, B.E., Spalding, D.B., 1974. The numerical computation of turbulent flows. Comput. Methods Appl. Mech. Engrg. 3, 269-289. 
